\title{
Exploring the gender gap in the labor market: A sex-disaggregated view
}

Jorge Zazueta ${ }^{\mathrm{a}}$ and Elvio Accinelli ${ }^{\mathrm{a}}$

${ }^{a}$ School of Ecnonomics, Universidad Autónoma de San Luis Potosí, México

\section{ARTICLE HISTORY}

Compiled November 12, 2020

\begin{abstract}
We develop a simple mathematical model describing the dynamics of the gender gap in a labor market niche, and study the effects of bias, market size and market dynamism in the evolution of the system. A high level characterization of the system is suggested by studying a large sample of the parameter space and specific cases of interest to policy making are explored.
\end{abstract}

\section{KEYWORDS}

Gender gap; labor market; market dynamism; dynamical system; equilibrium

\section{Introduction}

"For most of history, Anonymous was a woman" This quote attributed to feminist writer Virginia Woolf ${ }^{1}$ criticizes the invisibility that women have had in literature. This observation readily generalizes to the political, professional and social spheres, where women have historically struggled with the place they had been assigned in the world: home and family (see, for example: Schein (2001),Blackstone (2003), Heilman (2001) and Heilman (2012)). According to Women in a changing global economy: 1994 World Survey on the Role of Women in Development (United Nations, 1995):

The role of women in different fields of social work, in science, literature, technology and politics, among others was very limited in the past due to the general discrimination they experienced. And although they currently occupy a larger space, there are still structures contrary to their participation. The current role of women in development is the result of trends unleashed by changes in the enabling environment coupled with the nature of global economic change. Market forces and the policy choices underlying them have propelled women into a decisive position in much of the global and national economy. However, the changes have not been fast enough, are not sufficiently secure and are not occurring everywhere. To equalize and accelerate the process, policies should be adopted by governments, by enterprises and by women themselves to address the main obstacles.

More than two decades later, the outlook remains grim, with the Global Gender Gap Report 2020 (World Economic Forum, 2019) stating that "None of us will see gender parity in our lifetimes, and nor likely will many of our children". The same report points

CONTACT Jorge Zazueta. Email: jorge.zazueta@uaslp.mx

${ }^{1}$ This famous quote is actually an adaptation of the phrase "I would venture to guess that Anon, who wrote so many poems without signing them, was often a woman" from Virginia Woolf's 1929 essay: A Room from One's Own. 
out that one of the greatest challenges is in closing the gender gap in emerging roles such as cloud computing, engineering and Data and AI where women representation is only $12 \%, 15 \%$ and $26 \%$ respectively. While ensuring that women are better equipped to compete in the workforce is certainly critical, understanding the effect of the support provided by both men and woman to a more inclusive environment is paramount. This final point is the genesis of our paper. To what extent can women promote their inclusion in different areas of society? and how much support is needed from men to eliminate the gender gap in terms of participation in the labor market? While equal opportunity for equal capacity is the official policy in most organizations, in practice, role assignation is biased by the - often unstated - preferences of the establishment.

Clifton et al. (2019), built a compartmental dynamical system model to study the dynamics of professional ladder in terms of gender bias and homophily, capturing the evolution of gender distribution across hierarchical levels. Their focus is on the evolution throughout the hierarchy, rather than the behavior within a specific niche. Other models are more statistical in nature, Shaw and Stanton (2012) rely on Markov chains to study the "leaky pipeline" of an academic career, while Holman, Stuart-Fox, and Hauser (2018) study a vast number of academic publications listed in PubMed and arXiv data on $S T E M M^{2}$ related journal publications to estimate and predict the current author gender ratio, its rate of change, and when parity will be reached.

Our model attempts to show the impact that a supporting or opposing position from men or women towards inclusion has on the long term gender distribution in the workplace. Under the assumption of equally qualified men and women competing for the same role, the key drivers of heterogeneity are the carrying capacity of the labor market acting as a growth restriction of the unrepresented gender, the level of support each gender grants to the other and to itself and the rate at which jobs flow out of our niche under study, which we refer to as market dynamism. Notice that support and opposition are not necessarily features of different genders. Pervasive stereotypes, such as those of the "caring mother" or "devoted homemaker" affect the hiring decision making process of men and women alike. These biases can lead to negative feedback loops where women with hiring responsibilities - immersed in a male-dominated culture - tend to favor men in the selection process, perpetuating a culture of inequality of which they are themselves victims. On the other hand, it is possible to conceive a culture that is either neutral or supportive of women as rightful contributors that leads, in the long term, to closing the gender gap. The rest of this paper, develops and analyses an evolutionary model of such dynamics.

\section{The Model}

We assume a labor market niche with a given carrying capacity $K$ that recruits talent from a pool of male and female applicants with uniform sex distribution and identical talent. The labor market is integrated by $y$ males and $x$ females with fixed preferences and biases towards recruiting their own and opposite sex, determining the recruiting dynamics. Members of the labor market niche also leave due to a variety of reasons such as promotion, retirement or even death in proportion to a market dynamism constant $r_{i}$, specific to each sex $i$ as represented by the following dynamical system:

${ }^{2}$ Science, Technology, Engineering, Mathematics, and Medicine 


$$
\begin{aligned}
& \dot{x}(t)=\left(\lambda_{w w} x(t)+\lambda_{m w}(t) y\right)\left(1-\frac{x(t)+y(t)}{K}\right)-r_{x} x(t) \\
& \dot{y}(t)=\left(\lambda_{w m} x(t)+\lambda_{m m} y(t)\right)\left(1-\frac{x(t)+y(t)}{K}\right)-r_{y} y(t)
\end{aligned}
$$

Where $\lambda_{i j}$ represents the strength of support of sex $i$ for recruiting sex $j$, and the market dynamism $0 \leq r_{i} \leq 1$ is the speed at which sex $i$ leaving the niche and the dot over a function represents derivative with respect to time. We can simplify the system by selecting the units so that $K=1$ and by choosing the strength constants such that $0 \leq \lambda_{i j} \leq 1$ and $\lambda_{i k}+\lambda_{j k}=1$ for all $i, j$ and $k$ in $\{w, m\}$. This way we can interpret $\lambda_{i j}$ as proportions and reduce the number of parameters in system 1 by 3 .

Letting $\alpha=\lambda_{w w}$ and $\beta=\lambda_{m m}$, and omitting the explicit time dependence of the variables for notation simplicity, we can rewrite the system as:

$$
\begin{aligned}
& \dot{x}=(\alpha x+(1-\beta) y)(1-x-y)-r_{x} x \\
& \dot{y}=((1-\alpha) x+\beta y)(1-x-y)-r_{y} y
\end{aligned}
$$

Note that by our definition of $\alpha$ and $\beta$, preferences and biases from one sex over another need to be symmetrical and complete. That is, if women support women by $\alpha$, they necessarily support men by $1-\alpha$. Men behave similarly.

\section{General Model Dynamics}

Our working model as described by system 2 has a bifurcation at $r_{x}=r_{y}=0$. That is, in the absence of market dynamism there is a fixed point at the origin and a continuum of fixed points defined by the line $1-x-y=0$, as illustrated in Figure 1.

The line $y=x$ divides our domain of interest in two regions. Points above the line represent population ratios favoring men, while points below the line favor women. When $\alpha=\beta$, the direction fields are parallel to the line $y=x$, implying that a population that starts in one region, remains in that region. When $\alpha>\beta(\alpha<\beta)$, the direction field tilts to the right (left), allowing points to move from one region to another for sufficiently large differences. We will investigate this effect further in the discussion section.

When $r_{i} \neq 0$, the system has up to three fixed points at the intersection of the two hyperbolas defined by the right hand side of the equations:

$$
\begin{aligned}
& F=(\alpha x+(1-\beta) y)(1-x-y)-r_{x} x \\
& G=((1-\alpha) x+\beta y)(1-x-y)-r_{y} y
\end{aligned}
$$

The left-side panel in Figure 2 shows the direction field when $\alpha=.7, \beta=.65, r_{x}=$ .5 , and $r_{y}=.4$. and illustrates the fixed points as intersections of the two hyperbolas defined by equation 3 . There is a trivial fixed point at $(0,0)$ representing the case with population zero. This point is intuitively unstable as any perturbation will trigger a 

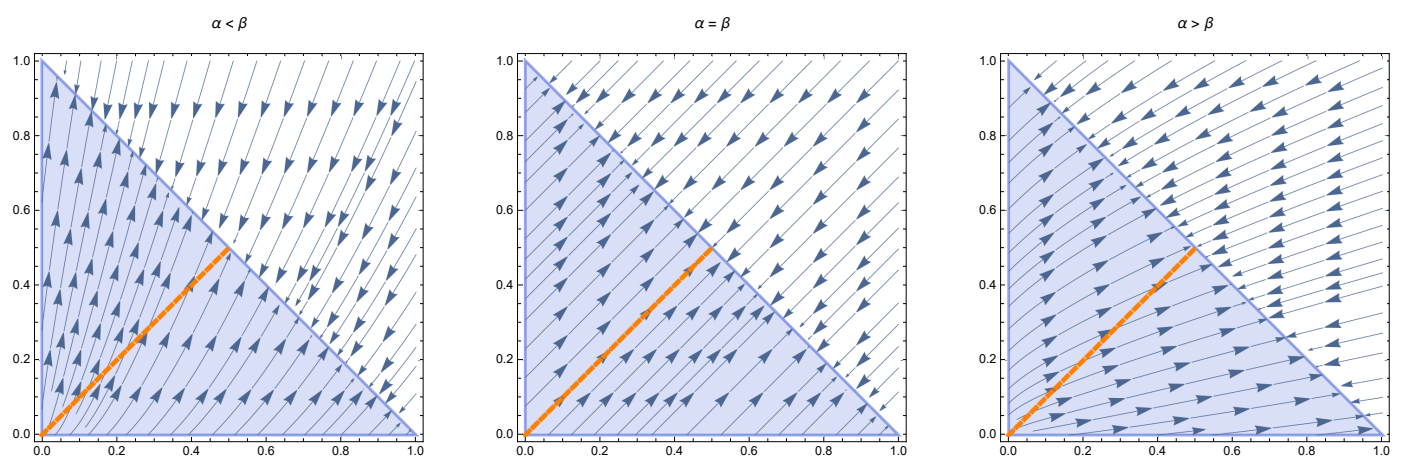

Figure 1. System Dynamics with no Market Dynamism. When $\alpha=\beta$ (central panel) the direction fields are parallel to the line $y=x$, tilting towards $y$ when $\alpha<\beta$, and $x$ when $\alpha>\beta$.

logistic-type population growth. The second point has positive, sub-unitary, values for both $x$ and $y$ representing the proportions of males and females with respect to the total population and the third point lies beyond our domain of interest.

Direction Field and Fixed Points

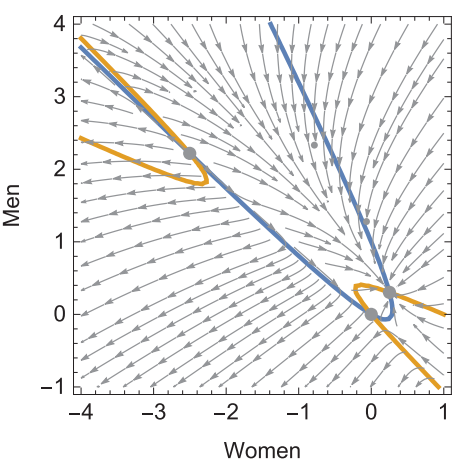

Women and Men Labor Market Dynamics

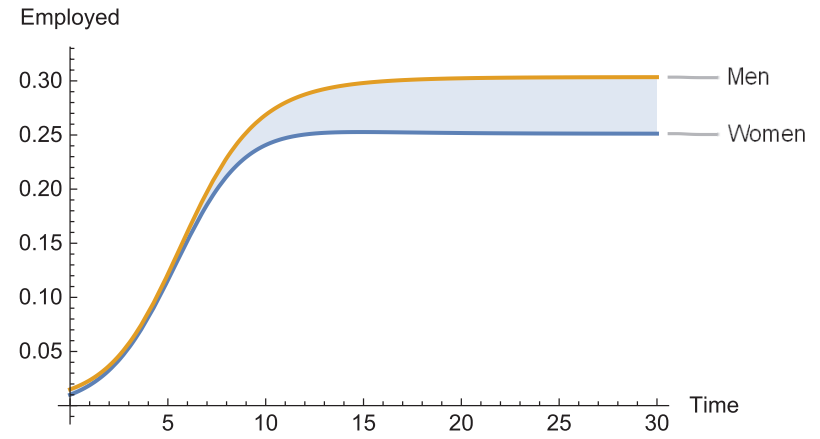

Figure 2. General system dynamics. The left-side panel shows the direction field and illustrates the fixed points as intersections of two hyperbolas for $\alpha=.7, \beta=.65, r_{x}=.5$, and $r_{y}=.4$. The right-hand side shows an instance of the evolution in time of the same system when $\left(x_{0}, y_{0}\right)=(.01, .015)$.

On the right side of the panel we can see how the gap between genders widens until the market is saturated and stabilizes with a mix that, in this particular case, (e.g. when $\left.\left(x_{0}, y_{0}\right)=(.01, .015)\right)$, favors men.

We are interested in characterizing the system dynamics across the parameter space defined as the set of 4 -tuples $\left(\alpha, \beta, r_{x}, r_{y}\right)$ whose entries take values in the closed unit interval.

To do this, we take a Lebesgue sample of size 5000 from the parameter space and, for each instance, we compute the eigenvalues of the associated Jacobian matrix evaluated at each of the corresponding fixed points. As depicted on Figure 3, we identified three consistent behaviors of the fixed points. The origin is always a, trivial, unstable fixed point with eigenvalues lying on the upper quadrants. An external fixed point appears either in the second or fourth quadrant leaving it out of our domain of interest, and lastly a principal fixed point appears not only in the first quadrant but within the triangle defined by the positive axis and the line $y=-x$. Its eigenvalues are always negative making it an attractor. 
Trivial

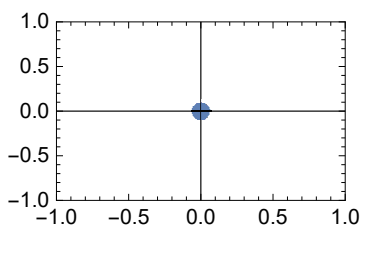

$\lambda_{T}$

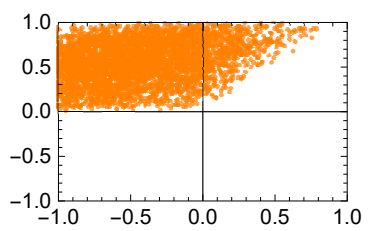

Principal

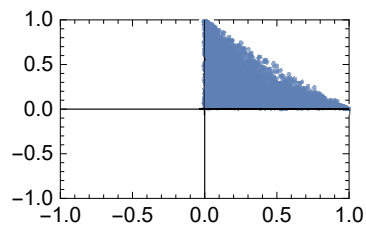

$\lambda_{P}$

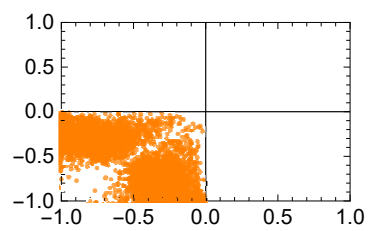

External
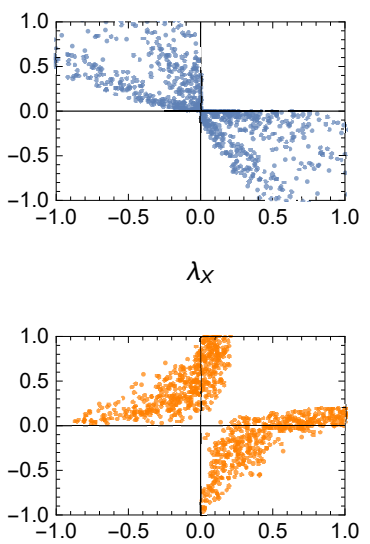

Figure 3. Fixed points and corresponding eigenvalues resulting from Lebesgue sampling the parameter space..

\section{Discussion}

Our main question is how can gender equality be achieved given initial conditions where males represent the majority of the population. The rest of the paper explores this question through a handful of scenarios.

\subsection{The Naïve Case}

We start by considering a setting without selection biases where every employee is hired based solely on its professional merits. Furthermore, employees do not leave their jobs during the period under study. Our system of equations is reduced to:

$$
\begin{aligned}
\dot{x} & =\frac{1}{2}(1-x-y)(x+y) \\
\dot{y} & =\frac{1}{2}(1-x-y)(x+y)
\end{aligned}
$$

The solutions for this simplified system can be found in closed form:

$$
\begin{aligned}
& y=\frac{e^{t}-c_{1} e^{t}-c_{1} e^{2 c_{2}}}{2\left(e^{t}+e^{2 c_{2}}\right)}+c_{1} \\
& x=\frac{e^{t}-c_{1} e^{t}-c_{1} e^{2 c_{2}}}{2\left(e^{t}+e^{2 c_{2}}\right)}
\end{aligned}
$$

Implying $y-x=c_{1}$ for any $t$. That is: a perfectly unbiased hiring environment, in the absence of market dynamism, preserves the current gender gap. Under the assumptions of our model, gap dynamics respond both to bias and market dynamism sometimes in not immediately obvious ways. We now introduce bias back into the system, while keeping market dynamism out. 


\subsection{Bias without market dynamism}

Reintroducing our bias parameters, $\alpha$ and $\beta$ our system becomes:

$$
\begin{aligned}
& \dot{x}=(\alpha x+(1-\beta) y)(1-x-y) \\
& \dot{y}=((1-\alpha) x+\beta y)(1-x-y)
\end{aligned}
$$

The fixed points are at the origin $(0,0)$ and along the line $\{(x, y) \mid x+y=1\}$. We restrict our attention to the region $[0,1] \times[0,1]$.

Direction Field and Fixed Points

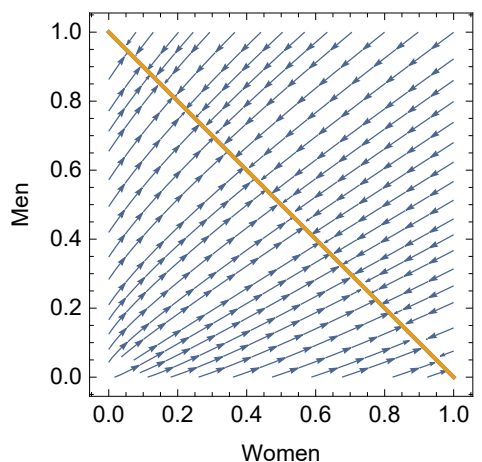

Women and Men Labor Market Behaviour

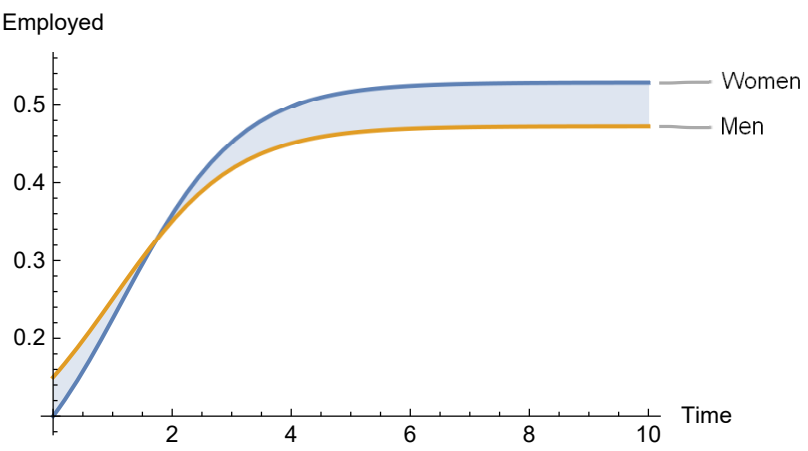

Figure 4. Bias without market dynamism. The left-hand side of the panel shows the direction fields moving towards the attractive line of fixed points. An instance of these dynamics where women overcome adverse initial conditions is depicted on the right-hand side.

As figure 4 suggests, for some values of $\alpha$ and $\beta$, the number of employed women can reach and surpass the number of men even when the initial conditions where unfavorable for women.-While our focus will be on the region below the fixed points line, the upper side can be interpreted as an overcrowded market that naturally corrects itself by subsiding towards the fixed-point line-That is, we are interested in parameter combinations such that the initially lower women curve, reaches or crosses the men curve. In principle, we should strive to identify parameters that result in an equality equilibrium, but this approach is impractical from a policy-making point of view where it would be more efficient to identify reasonable policies that increase the job generation speed for women and readjust once equity is achieved. To simplify the analysis, we define the gender gap

$$
\gamma\left(t, \alpha, \beta, x_{0}, y_{0}\right)=y\left(t, \alpha, \beta, x_{0}, y_{0}\right)-x\left(t, \alpha, \beta, x_{0}, y_{0}\right)
$$

as the difference between the number of employed men and women, and study the cases when it crosses the $x$ axis. Figure 5 illustrates the behavior of the gap curve for a range of values of $\beta$ when $\alpha$ is chosen to exceed it by five percent. Note that depending on the value of $\beta$ this difference might of might not be sufficient to close the gap.

To better understand the sensitivity of the gap with respect to the ratio of $\alpha$ and $\beta$ given the initial conditions $x(0)=x_{0}$ and $y(0)=y_{0}$, we define the function $E$ as 


\section{Gap dynamics for different values of $\beta$}

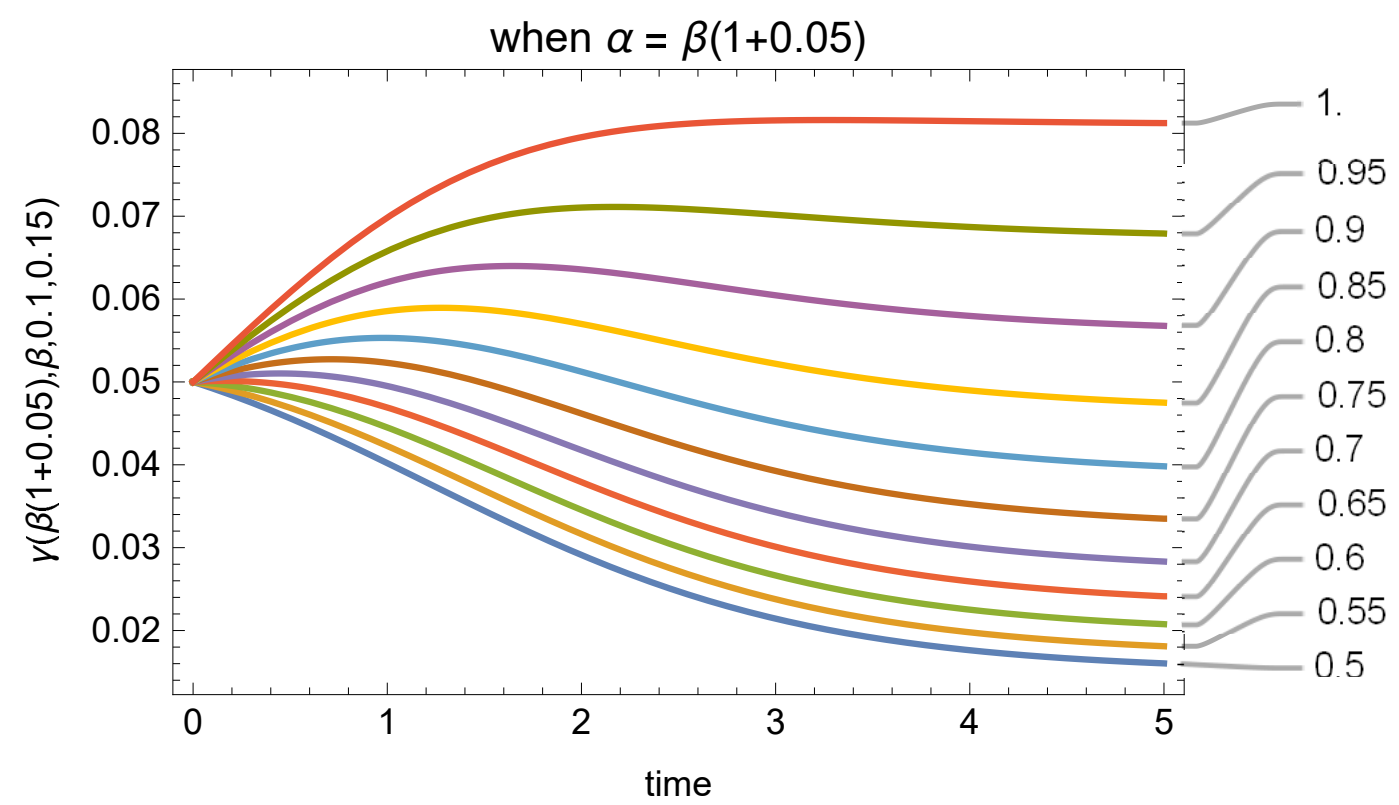

Figure 5. Gap dynamics. As the value of $\beta$ increases, $\alpha$ fails to be large enough to close the gap.

the minimum increment $\epsilon$ of $\alpha$ with respect to $\beta$ required to make the gap cross the $x$ axis.

$$
E\left(\beta, x_{0}, y_{0}\right)=\min \left\{\epsilon \mid \gamma\left(t, \beta(1+\epsilon), \beta, x_{0}, y_{0}\right)=0, \text { for some } t \text { in } \Re^{+}\right\}
$$

Figure 6 illustrates the behavior of $E$ for various initial conditions. The panel on the left shows how an increase in the absolute values of the initial population while preserving their proportion (in this case $\frac{x_{0}}{y_{0}}=\frac{2}{3}$ ) increases the minimum difference requirement for achieving equality. In light of our unit normalization to set market capacity equal to one, the increase in the value of initial conditions can be interpreted as a decrease in the relative market size. Thus, given equal initial conditions, the required change in $\alpha$ increases in the opposite direction of market capacity, suggesting that a healthy, growing labor market is more conducive to gender equality under the same preference and bias assumptions. As the market saturates, an equilibrium is reached and no change in preferences, either natural or induced, will have an effect on the population proportion. As $\beta$ moves from $\frac{1}{2}$ to $1, E$ increases, somewhat slowly until it reaches the constraint $E=1-\beta$, thus shrinking the policy range as the market saturates. The panel on the right shows how increasing initial disparity poses a challenge to equality, with a reducing policy range. In the highest scenario with an initial population ratio of $\frac{10}{25}$ - which is not unrealistic - there are no policy options for $\beta>\frac{1}{2}$ even in a relatively large market size. The cases where $\beta<\frac{1}{2}$ represent the somewhat rare cases where men show preference for hiring women. Increasing the rate of change of hired women, requiring that women show a rapidly increased preference for men to compensate. 
Minimum increase with respect to $\beta$ to

reach equality as market size decreases

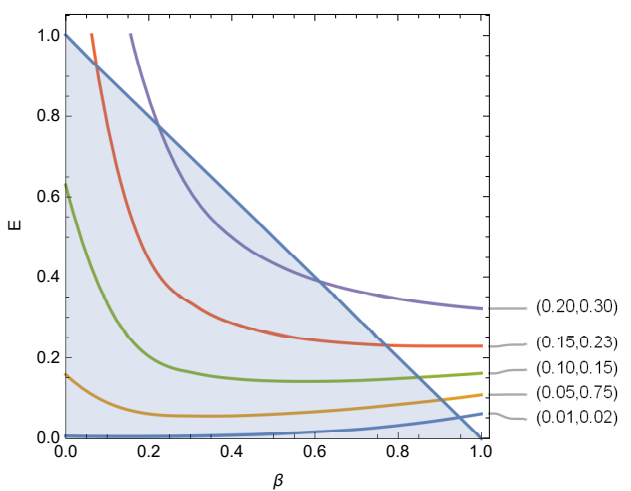

Minimum increase with respect to $\beta$ to reach equality as the initial population proportion changes

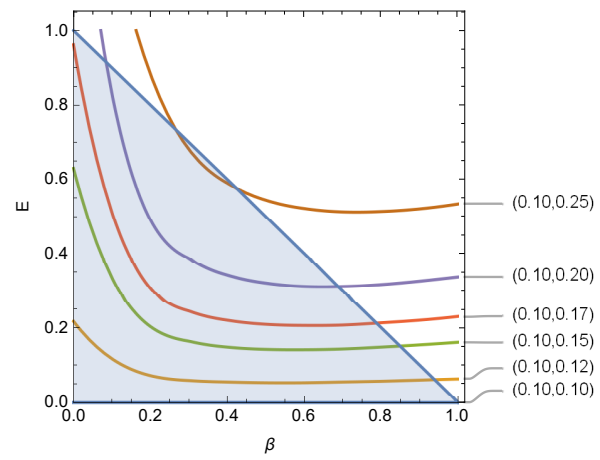

Figure 6. Required change in women's preferences to push gender equality as a function of male bias.

\subsection{The effect of market dynamism}

In the absence of market dynamism we saw that an existing gap can only be closed by introducing bias into the system, and that outside of a tight window, the required bias might be too large to be practical. Market dynamism enriches the dynamics of the system by allowing employees to flow out of their niche leaving an open spot to be occupied by someone else. We return to the general system of equations 2 , and turn our attention first to the case where $r_{x}=r_{y}=r$. This allow us to examine the intrinsic behavior of the system without the pull of an external dynamic.

Direction Field and Fixed Points

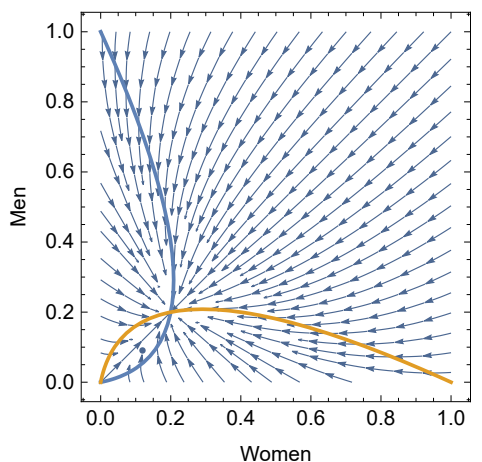

Women and Men Labor Market Behaviour

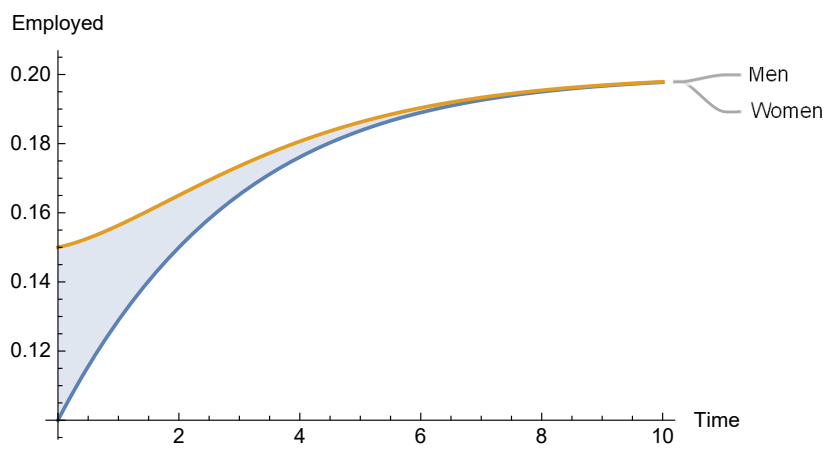

Figure 7. No bias with equal market dynamism. By introducing market dynamism, a non biased niche can reach gender equity naturally.

$$
\begin{aligned}
& \dot{x}=(\alpha x+(1-\beta) y)(1-x-y)-r x \\
& \dot{y}=((1-\alpha) x+\beta y)(1-x-y)-r y
\end{aligned}
$$

From figure 7 , we see that market dynamism can drive equality on an unbiased 
niche even when the initial conditions are unfavorable to women. Furthermore, when $\alpha=\beta$, and $r_{x}=r_{y}=r \neq 0$ the principal fixed point becomes $\left(\frac{1-r}{2}, \frac{1-r}{2}\right)$, making equality an attractor, that varies across the line $y=x$ in proportion to $r$.

Direction Field and Fixed Points

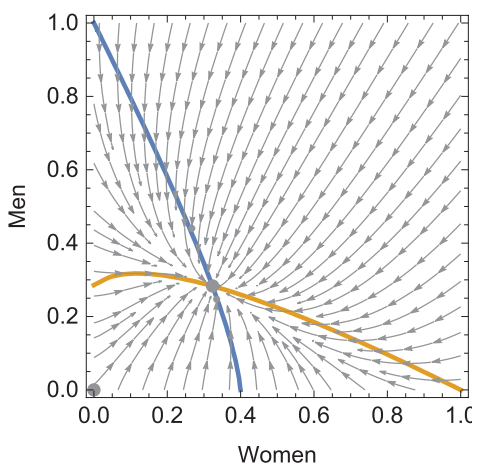

Women and Men Labor Market Dynamics

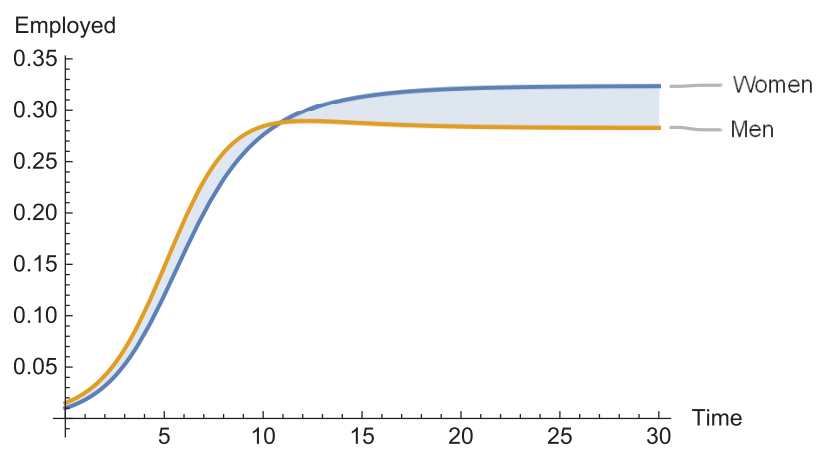

Figure 8. Asymmetric market dynamism can mask niche dynamics. In the right hand side of the panel $\alpha=0.5, \beta=0.7, r_{x}=0.3$, and $r_{y}=0.5$.

While market dynamism can play an equalizing role by increasing the speed by which the system state changes, asymmetric dynamism $\left(\right.$ i.e.,$\left.r_{x} \neq r_{y}\right)$ can mask our perception of local dynamics. As figure 8 illustrates, an equilibrium favoring women can result from a man-biased system with a market dynamism that speeds up the outflow of men out of the niche. A possible interpretation is a market where men are promoted up more frequently than women, and the resulting niche-majority is a reflection not of a favorable endogenous dynamic, but a result of a adverse exogenous one. This last example stresses the importance of understanding the dynamics of a system before attempting to measure it.

\section{Conclusions}

We developed a simple mathematical model to describe the gender dynamics within a labor market niche in terms of sex-disaggregated bias, the carrying capacity of the niche and market dynamism (see equation 2). Our system presents a bifurcation when market dynamism is zero, in which case we have an unstable fixed point at zero and a continuum of stable fixed points across the line $x+y=1$. One of the implication being that in the absence of market dynamism, a perfectly unbiased hiring environment (i.e., $\alpha=\beta=\frac{1}{2}$ ) preserves the existing gender gap. A natural question is then how much bias needs to be introduced into the system (perhaps via policy or an incentive program) to close the prevailing gap.

We explored this situation by looking at the requisite change in women preferences $(\alpha)$ given equal initial conditions, finding that it increases in the opposite direction of market capacity, suggesting that a healthy, growing labor market is more conducive to gender equality under the same preference and bias assumptions. As the market saturates, an equilibrium is reached and no change in preferences, either natural or induced, will have an effect on the population proportion. As these proportions become less favorable, the policy range might shrink out of scope even with a relatively large market. Not surprisingly, increasingly unfavorable initial conditions also reduce the 
scope of the policy range.

When market dynamism is reintroduced $\left(r_{x}, r_{y} \neq 0\right)$, our system has three fixed points. The trivial one at the origin, and "external one" that lies out of our domain of interest as it implies negative population proportions and "principal" stable fixed point within the triangle defined by the points in the first quadrant that lie below the line $x+y=1$. In the case of symmetric market dynamism $\left(r_{x}=r_{y}=r\right)$ we found that it can drive equity in an unbiased environment even when the initial conditions favor one sex. Furthermore, as long as both sexes discriminate equally, a large enough market dynamism can drive equity in the long run.

Thus, the gender gap depends not only on biases and preferences of the market players, but on market size and dynamism. These exogenous characteristics have a significant effect on the market equilibrium and can introduce unexpected behavior. In particular, asymmetric market dynamism can induce perceived equity or inequity on a given niche masking its intrinsic dynamics. This has implications for those attempting to measure the configuration of a market niche without knowledge of how it is being influence by exogenous dynamics.

\section{References}

Blackstone, A. M. (2003). Gender roles and society [Book Section]. In J. R. Miller, R. M. Lerner, \& L. B. Schiamberg (Eds.), Human ecology: An encyclopedia of children, families, communities, and environments (p. 335-338). Santa Barbara, CA: University of Maine.

Clifton, S. M., Hill, K., Karamchandani, A. J., Autry, E. A., McMahon, P., \& Sun, G. (2019). Mathematical model of gender bias and homophily in professional hierarchies [Journal Article]. Chaos: An Interdisciplinary Journal of Nonlinear Science, 29(2), 023135. Retrieved from https://aip.scitation.org/doi/abs/10.1063/1.5066450

Heilman, M. E. (2001). Description and prescription: How gender stereotypes prevent women's ascent up the organizational ladder. Journal of Social Issues, 57(4), 657-674.

Heilman, M. E. (2012). Gender stereotypes and workplace bias [Journal Article]. Research in Organizational Behavior, 32, 113-135. Retrieved from https://dx.doi.org/10.1016/j.riob.2012.11.003

Holman, L., Stuart-Fox, D., \& Hauser, C. E. (2018). The gender gap in science: How long until women are equally represented? [Journal Article]. PLOS Biology, 16(4), e2004956. Retrieved from https://dx.doi.org/10.1371/journal.pbio. 2004956

Schein, V. E. (2001). A global look at psychological barriers to women's progress in management [Journal Article]. Journal of Social Issues, 57(4), 675-688. Retrieved from https://dx.doi.org/10.1111/0022-4537.00235

Shaw, A. K., \& Stanton, D. E. (2012). Leaks in the pipeline: separating demographic inertia from ongoing gender differences in academia [Journal Article]. Proceedings of the Royal Society B: Biological Sciences, 279(1743), 3736-3741. Retrieved from https://dx.doi.org/10.1098/rspb.2012.0822

United Nations. (1995). Women in a changing global economy : 1994 world survey on the role of women in development. (Report). United Nations. Retrieved from https://digitallibrary.un.org/record/207546? ln=en

World Economic Forum. (2019). Global gender gap report 2020 (Report). Retrieved from https://www. weforum. org/reports/gender-gap-2020-report-100-years-pay-equality 